\title{
Direct, Catalytic Enantioselective Nitroaldol (Henry) Reaction of Trifluoromethyl Ketones: An Asymmetric Entry to a-Trifluoromethyl-substituted Quaternary Carbons
}

\author{
Fernando Tur and José M. Saá* \\ Departament de Química, Universitat de les Illes Balears, 07122 Palma de Mallorca (Spain) \\ jmsaa@uib.es
}

\section{Supporting information}

\section{Table of contents:}

A) General remarks. S2

B) General procedure for the preparation of lanthanum (III) triflate salt complex $[(\triangle, S, S, S)$ Binolam $]_{3} \cdot \operatorname{La}(\mathrm{OTf})_{3}$ 2La. S2

C) General procedure for the synthesis of trifluoromethyl ketones $\mathbf{3 f}-\mathbf{g}$. S2-S3

D) General procedure for the catalytic asymmetric nitroaldol reaction of trifluoromethyl ketones 3a-g. S3

E) General procedure for the "enantiomer self-disproportionation test". S5

F) General procedure for the reduction of $\alpha$-trifluoromethyl tertiary nitroaldols 4 to the corresponding $\beta$-amino- $\alpha$-trifluoromethyl tertiary alcohols 5 . S6

G) Attempted kinetic resolution of racemic nitroaldol 4c. S7

$\mathrm{H})$ Evaluation of retro-nitroaldol reaction upon enantiomerically enriched $\mathbf{4 c}$. S8

I) General procedure for the synthesis of racemic $\alpha$-trifluoromethyl tertiary nitroaldols 4ag. S8

J) Assignment of the absolute configuration of adduct 5c. S8-S13 


\section{A) General remarks:}

Commercial (Sigma-Aldrich Co.) lanthanum (III) triflate of the highest quality available (99.999\%) was dried under vacuum for $96 \mathrm{~h}$ at $220^{\circ} \mathrm{C}$ in a Büchi Glass Oven B-580 and stored in a dessicator. Prior to its use for the preparation of the catalyst (see below) the appropriate amount was dried under vacuum for an extra $2 \mathrm{~h}$ at $220^{\circ} \mathrm{C}$. Trifluoromethyl ketones 3a-e were obtained from commercial sources, and were used as received. Trifluoromethyl ketones $\mathbf{3 f}-\mathbf{g}$ were synthesized following the reported procedure. ${ }^{1}$ Nitromethane was obtained from commercial sources, and was used as received. Commercial grade solvents were boiled with the appropriate drying agent, and eventually distilled prior to use. ${ }^{2}$ Analytical thin layer chromatography was performed on Fluka Polygram Sil G/UV ${ }_{254}$ plates, whose visualization was accomplished under UV light or under iodine vapors. Purification of reaction products was carried out by column chromatography using Merck silica gel 60 (70-230 mesh). Melting points were measured on a Büchi Dr. Tottoli apparatus and are uncorrected. Optical rotations were measured on a Perkin Elmer 341 LC polarimeter provided with a sodium lamp and are reported as follows: $[\alpha]^{\mathrm{T}\left({ }^{\circ} \mathrm{C}\right)}(\mathrm{c}=\mathrm{g} / 100 \mathrm{~mL}$, solvent). Infrared spectra were recorded on a Nicolet Impact 400 spectrometer and are reported in wavenumbers $\left(\mathrm{cm}^{-1}\right)$. Enantiomeric excesses (ee) were determined by chiral HPLC analysis using a Waters chromatograph provided with Waters 600E System Controller, Waters Tunable Absorbance Detector 486 and Daicel Chiralpak AS-H or Chiralcel OJ-H columns (see below for further details). ${ }^{1} \mathrm{H}$ NMR spectra were recorded on a Bruker AMX-300 (300 MHz) spectrometer and are reported in ppm, using solvent as internal standard $\left(\mathrm{CDCl}_{3}\right.$ calibrated at $\left.7.26 \mathrm{ppm}\right)$. Data are reported either as: $\mathrm{s}=$ singlet, $\mathrm{d}=$ doublet, $\mathrm{dd}$ $=$ double doublet, $\mathrm{t}=$ triplet, $\mathrm{q}=$ quartet, $\mathrm{m}=$ multiplet, $\mathrm{br}=$ broad, coupling constant $(\mathrm{s})$ in $\mathrm{Hz}$, integration. Proton-decoupled ${ }^{13} \mathrm{C}$ NMR spectra were recorded on a Bruker AMX-300 (75.5 $\mathrm{MHz})$ spectrometer and are reported in ppm using solvent as an internal standard $\left(\mathrm{CDCl}_{3}\right.$ calibrated at $77.16 \mathrm{ppm}$ ). Proton-decoupled ${ }^{19} \mathrm{~F}$ NMR were recorded on a Bruker AMX-300 $(282 \mathrm{MHz})$ spectrometer and are reported in ppm using $\mathrm{CF}_{3} \mathrm{COOH}$ as external reference calibrated at $-76.55 \mathrm{ppm}$. Low and high resolution mass spectra by use either electronic impact ionization $\left(\mathrm{EI}^{+}\right)$or fast atom bombardment ionization $\left(\mathrm{FAB}^{+}\right)$were obtained on a Micromass Autospect spectrometer. Low and high resolution mass spectra by use of electrospray ionization $\left(\mathrm{ESI}^{+}\right)$were obtained on a Bruker Microtof spectrometer. X-ray data were collected on a Bruker Nonius FR591 KappaCCD2000 diffractometer at $100 \mathrm{~K}$.

\section{B) General procedure for the preparation of lanthanum (III) triflate salt complex $[(\Delta, S, S, S) \text {-Binolam }]_{3} \cdot \mathrm{La}(\mathrm{OTf})_{3} 2 \mathrm{La}$ :}

2La has been prepared as reported by Saá, J. M.; Tur, F.; González, J.; Vega, M. Tetrahedron: Asymmetry 2006, 17, 99-106.

\section{C) General procedure for the synthesis of trifluoromethyl ketones $3 \mathrm{f}-\mathrm{g}:{ }^{1}$}

A commercial $1 \mathrm{M}$ solution of the appropriate Grignard reagent in $\mathrm{Et}_{2} \mathrm{O}$ (or THF) (30 $\mathrm{mL}, 0.03 \mathrm{~mol}, 1$ equiv.) was added dropwise to a stirred solution of ethyl trifluoroacetate $(4 \mathrm{~mL}$, $0.033 \mathrm{~mol}, 1.1$ equiv.) in $50 \mathrm{~mL}$ of $\mathrm{Et}_{2} \mathrm{O}$ held at $-78^{\circ} \mathrm{C}$ in a dry ice-acetone bath. On completion of the addition, the stirred mixture was allowed to warm to room temperature. Aqueous $\mathrm{NH}_{4} \mathrm{Cl}$ solution $(25 \mathrm{~mL})$ was added followed by dilute $\mathrm{HCl}(25 \mathrm{~mL})$. The organic phase was separated, washed with saturated $\mathrm{NaCl}$ solution, and dried over $\mathrm{MgSO}_{4}$. Solvents were removed under reduced pressure affording trifluoromethyl ketones $\mathbf{3 f}-\mathbf{g}$.

1-(4-tert-butyl-phenyl)-2,2,2-trifluoro-ethanone (3f): The crude product $3 \mathbf{f}$ was purified by column chromatography (silica gel, $n$-hexane/ $\mathrm{Et}_{2} \mathrm{O} 9: 1$ ) thereby affording $\mathbf{3 f}$ as a pale yellow solid. Yield: $94 \%$. The product was positively identified as $\mathbf{3 f}$ by comparison of its spectral data with that reported in the literature. ${ }^{3}$ 
1,1,1-trifluoro-4-phenyl-but-3-yn-2-one (3g): The crude product $\mathbf{3 g}$ was purified by distillation under reduced pressure $\left(\mathrm{T}=95-100{ }^{\circ} \mathrm{C}\right)$ thereby affording $\mathbf{3 g}$ as a yellow oil. Yield: $35 \%$. The product was positively identified as $\mathbf{3} \mathbf{g}$ by comparison of its spectral data with that reported in the literature. ${ }^{4}$

\section{D) General procedure for the catalytic asymmetric nitroaldol reaction of trifluoromethyl ketones 3a-g:}

To a solution of $[(\Delta, S, S, S) \text {-Binolam }]_{3} \cdot \mathrm{La}(\mathrm{OTf})_{3} \mathbf{2} \mathbf{L a}(260 \mathrm{mg}, 0.1325 \mathrm{mmol}, 25 \mathrm{~mol} \%$, 0.25 equiv. $)$ in dry acetonitrile $(5 \mathrm{~mL})$, at $-40{ }^{\circ} \mathrm{C}$, under argon, proton sponge ${ }^{\circledR}(475 \mu \mathrm{L}, 0.1325$ $\mathrm{mmol}, 25 \mathrm{~mol} \%, 0.25$ equiv., $280 \mathrm{mM}$ solution in dry acetonitrile) was added. After stirring for $5 \mathrm{~min}$. the corresponding trifluoromethyl ketone 3a-g $(0.53 \mathrm{mmol}, 1$ equiv.) and excess nitromethane ( $291 \mu \mathrm{L}, 5.3 \mathrm{mmol}, 10$ equiv.) were added and the mixture stirred for $96 \mathrm{~h}$. The reaction mixture was then quenched with the appropriate amount of TFA $(0.265 \mathrm{mmol}, 0.50$ equiv.) followed by evaporation to dryness. The crude mixture was purified by column chromatography (silica gel, $\mathrm{CHCl}_{3}$ ) thereby affording $\alpha$-trifluoromethyl tertiary nitroaldols $\mathbf{4 a - g}$.

\section{(S)-1,1,1-trifluoro-2-nitromethyl-butan-2-ol (4a):}<smiles>CCC(O)(CC)C[N+](=O)[O-]</smiles>

4a The title compound 4a was prepared according to the general procedure described in section D. Yield: $55 \%$; pale yellow oil; ${ }^{1} \mathrm{H}$ NMR $\left(300 \mathrm{MHz}, \mathrm{CDCl}_{3}\right) \delta: 4.65(\mathrm{~d}, J=$ $12.6 \mathrm{~Hz}, 1 \mathrm{H}), 4.57(\mathrm{~d}, J=12.9 \mathrm{~Hz}, 1 \mathrm{H}), 4.02(\mathrm{~s}, 1 \mathrm{H}), 1.97-1.73(\mathrm{~m}, 2 \mathrm{H}), 1.03(\mathrm{t}, J=7.5 \mathrm{~Hz}$, $3 \mathrm{H}) ;{ }^{13} \mathrm{C}$ NMR $\left(75.5 \mathrm{MHz}, \mathrm{CDCl}_{3}\right) \delta: 124.8\left(\mathrm{q},{ }^{1} J_{\mathrm{C}-\mathrm{F}}=287.1 \mathrm{~Hz}\right), 76.2,75.1\left(\mathrm{q},{ }^{2} J_{\mathrm{C}-\mathrm{F}}=28.5 \mathrm{~Hz}\right)$, 26.0, 6.7; ${ }^{19} \mathrm{~F}$ NMR $\left(282 \mathrm{MHz}, \mathrm{CDCl}_{3}\right) \delta:-79.3$ (s, 3F); HRMS (EI ${ }^{+}-\mathrm{TOF}$ ): exact mass calcd. for $\mathrm{C}_{3} \mathrm{H}_{3} \mathrm{~F}_{3} \mathrm{NO}_{3}\left[\mathrm{M}-\mathrm{C}_{2} \mathrm{H}_{5}\right]^{+}:$158.0065, found 158.0058; the enantiomeric purity of 4a was determined by chiral HPLC analysis using a Daicel Chiralpak AS-H column; $n$-hexane/2propanol $95: 5 ; 1 \mathrm{~mL} / \mathrm{min} ; \lambda=215 \mathrm{~nm} . t_{\mathrm{R}}($ minor $)=11.1 \mathrm{~min} ; t_{\mathrm{R}}($ major $)=12.0 \mathrm{~min} ; 85 \%$ ee.

(S)-1,1,1-trifluoro-2-nitromethyl-3-phenyl-propan-2-ol (4b):<smiles>O=[N+]([O-])CC(O)(Cc1ccccc1)C(F)(F)F</smiles>

The title compound $\mathbf{4 b}$ was prepared according to the general procedure described in section D. Yield: 93\%; pale yellow oil; ${ }^{1} \mathrm{H}$ NMR $\left(300 \mathrm{MHz}, \mathrm{CDCl}_{3}\right) \delta$ : 7.40-7.29 $(\mathrm{m}, 5 \mathrm{H}), 4.65(\mathrm{~d}, J=13.1 \mathrm{~Hz}, 1 \mathrm{H}), 4.65(\mathrm{br} \mathrm{s}, 1 \mathrm{H}), 4.26(\mathrm{dd}, J=13.1,1 \mathrm{~Hz}), 3.31(\mathrm{~d}, J=14.3$ $\mathrm{Hz}, 1 \mathrm{H}), 2.95(\mathrm{~d}, J=14.5 \mathrm{~Hz}, 1 \mathrm{H}) ;{ }^{13} \mathrm{C}$ NMR $\left(75.5 \mathrm{MHz}, \mathrm{CDCl}_{3}\right) \delta: 132.1,131.0,129.0,128.2$, $124.6\left(\mathrm{q},{ }^{1} J_{\mathrm{C}-\mathrm{F}}=287.1 \mathrm{~Hz}\right), 75.9,74.9\left(\mathrm{q},{ }^{2} J_{\mathrm{C}-\mathrm{F}}=28.5 \mathrm{~Hz}\right), 38.4 ;{ }^{19} \mathrm{~F} \mathrm{NMR}\left(282 \mathrm{MHz}, \mathrm{CDCl}_{3}\right) \delta$ : -80.4 (s, 3F); HRMS (EI ${ }^{+}$-TOF): exact mass calcd. for $\mathrm{C}_{10} \mathrm{H}_{10} \mathrm{~F}_{3} \mathrm{NO}_{3}[\mathrm{M}]^{+}: 249.0613$, found 249.0618; the enantiomeric purity of $\mathbf{4 b}$ was determined by chiral HPLC analysis using a Daicel Chiralcel OJ-H column; $n$-hexane/2-propanol 9:1; $1 \mathrm{~mL} / \mathrm{min} ; \lambda=215 \mathrm{~nm} . t_{\mathrm{R}}$ (major) $=10.4$ $\min ; t_{\mathrm{R}}($ minor $)=12.6 \mathrm{~min} ; 92 \%$ ee.

\section{(S)-1,1,1-trifluoro-3-nitro-2-phenyl-propan-2-ol $(4 \mathrm{c}):{ }^{5}$}<smiles>O=[N+]([O-])CC(O)(c1ccccc1)C(F)(F)F</smiles>

The title compound $\mathbf{4 c}$ was prepared according to the general procedure described in section D. Yield: 78\%; pale yellow solid; mp: $47-49{ }^{\circ} \mathrm{C}$; $[\alpha]^{25}{ }_{\mathrm{D}}+41.8^{\circ}$ (c 1.7, $\mathrm{MeOH}) ;{ }^{1} \mathrm{H}$ NMR $\left(300 \mathrm{MHz}, \mathrm{CDCl}_{3}\right) \delta: 7.64-7.61(\mathrm{~m}, 2 \mathrm{H}), 7.46-7.43(\mathrm{~m}, 3 \mathrm{H}), 5.12(\mathrm{~d}, J=13.8$ $\mathrm{Hz}, 1 \mathrm{H}), 5.03(\mathrm{~d}, J=13.5 \mathrm{~Hz}, 1 \mathrm{H}), 4.79(\mathrm{~s}, 1 \mathrm{H}) ;{ }^{13} \mathrm{C} \mathrm{NMR}\left(75.5 \mathrm{MHz}, \mathrm{CDCl}_{3}\right) \delta: 133.2,130.2$, $129.1,126.3,123.6\left(\mathrm{q},{ }^{1} J_{\mathrm{C}-\mathrm{F}}=286 \mathrm{~Hz}\right), 77.7,76.4\left(\mathrm{q},{ }^{2} J_{\mathrm{C}-\mathrm{F}}=30.2 \mathrm{~Hz}\right) ;{ }^{19} \mathrm{~F}$ NMR $(282 \mathrm{MHz}$, 
$\left.\mathrm{CDCl}_{3}\right) \delta$ : -79.0 (s, 3F); IR (KBr)v: 3499, 1379; HRMS (EI+-TOF): exact mass calcd. for $\mathrm{C}_{9} \mathrm{H}_{8} \mathrm{~F}_{3} \mathrm{NO}_{3}[\mathrm{M}]^{+}: 235.0456$, found 235.0454; the enantiomeric purity of $\mathbf{4 c}$ was determined by chiral HPLC analysis using a Daicel Chiralcel OJ-H column; $n$-hexane/2-propanol 9:1; 1 $\mathrm{mL} / \mathrm{min} ; \lambda=215 \mathrm{~nm} . t_{\mathrm{R}}$ (major) $=33.8 \mathrm{~min} ; t_{\mathrm{R}}($ minor $)=37.9 \mathrm{~min} ; 96 \%$ ee.

(S)-1,1,1-trifluoro-3-nitro-2-(3-trifluoromethyl-phenyl)-propan-2-ol (4d):<smiles>O=[N+]([O-])CC(O)(c1cccc(C(F)(F)F)c1)C(F)(F)F</smiles>

The title compound $\mathbf{4 d}$ was prepared according to the general procedure described in section D. Yield: 55\%; pale yellow oil; ${ }^{1} \mathrm{H}$ NMR $\left(300 \mathrm{MHz}, \mathrm{CDCl}_{3}\right) \delta: 7.93(\mathrm{~s}, 1 \mathrm{H})$, 7.79-7.71 (m, 2H), $7.587(\mathrm{t}, J=7.8 \mathrm{~Hz}, 1 \mathrm{H}), 5.11(\mathrm{~d}, J=13.5 \mathrm{~Hz}, 1 \mathrm{H}), 5.05(\mathrm{~d}, J=13.5 \mathrm{~Hz}$, $1 \mathrm{H}), 4.85(\mathrm{~s}, 1 \mathrm{H}) ;{ }^{13} \mathrm{C}$ NMR $\left(75.5 \mathrm{MHz}, \mathrm{CDCl}_{3}\right) \delta: 134.3,132.0\left(\mathrm{q},{ }^{3} J_{\mathrm{C}-\mathrm{F}}=3 \mathrm{~Hz}\right), 129.7,129.6$, $127.0\left(\mathrm{q},{ }^{3} J_{\mathrm{C}-\mathrm{F}}=3.8 \mathrm{~Hz}\right), 123.7\left(\mathrm{q},{ }^{1} J_{\mathrm{C}-\mathrm{F}}=272.5 \mathrm{~Hz}\right), 123.5\left(\mathrm{~d},{ }^{3} J_{\mathrm{C}-\mathrm{F}}=2.3 \mathrm{~Hz}\right), 123.3\left(\mathrm{q},{ }^{1} J_{\mathrm{C}-\mathrm{F}}=\right.$ $285.4 \mathrm{~Hz}), 77.5,75.5\left(\mathrm{q},{ }^{2} J_{\mathrm{C}-\mathrm{F}}=28 \mathrm{~Hz}\right) ;{ }^{19} \mathrm{~F}$ NMR $\left(282 \mathrm{MHz}, \mathrm{CDCl}_{3}\right) \delta:-63.5(\mathrm{~s}, 3 \mathrm{~F}),-79.3(\mathrm{~s}$, 3F); HRMS (EI ${ }^{+}-\mathrm{TOF}$ ): exact mass calcd. for $\mathrm{C}_{9} \mathrm{H}_{8} \mathrm{~F}_{3} \mathrm{NO}_{3}[\mathrm{M}]^{+}: 235.0456$, found 235.0454; the enantiomeric purity of $\mathbf{4 c}$ was determined by chiral HPLC analysis using a Daicel Chiralcel OJ$\mathrm{H}$ column; $n$-hexane/2-propanol 9:1; $1 \mathrm{~mL} / \mathrm{min} ; \lambda=215 \mathrm{~nm}$. $t_{\mathrm{R}}($ major $)=12.7 \mathrm{~min} ; t_{\mathrm{R}}(\operatorname{minor})=$ $15.1 \mathrm{~min} ; 67 \%$ ee.

(S)-1,1,1-trifluoro-2-(4-fluoro-phenyl)-3-nitro-propan-2-ol (4e):<smiles>O=[N+]([O-])CC(O)(c1ccc(F)cc1)C(F)(F)F</smiles>
described in section D. Yield: $68 \%$; pale yellow solid; mp: $41-43{ }^{\circ} \mathrm{C} ;[\alpha]^{25}{ }_{\mathrm{D}}+15^{\circ}\left(\mathrm{c} 0.8, \mathrm{CHCl}_{3}\right)$; ${ }^{1} \mathrm{H}$ NMR $\left(300 \mathrm{MHz}, \mathrm{CDCl}_{3}\right) \delta$ : 7.61-7.56 (m, 2H), 7.17-7.11 (m, 2H), $5.07(\mathrm{~d}, J=13.8 \mathrm{~Hz}, 1 \mathrm{H})$, $4.97(\mathrm{~d}, J=13.5 \mathrm{~Hz}, 1 \mathrm{H}), 4.66(\mathrm{~s}, 1 \mathrm{H}) ;{ }^{13} \mathrm{C} \mathrm{NMR}\left(75.5 \mathrm{MHz}, \mathrm{CDCl}_{3}\right) \delta: 163.8\left(\mathrm{~d},{ }^{1} J_{\mathrm{C}-\mathrm{F}}=250.3\right.$ $\mathrm{Hz}), 128.9\left(\mathrm{~d},{ }^{3} J_{\mathrm{C}-\mathrm{F}}=3.8 \mathrm{~Hz}\right), 128.5\left(\mathrm{~d},{ }^{3} J_{\mathrm{C}-\mathrm{F}}=8.8 \mathrm{~Hz}\right), 116.2\left(\mathrm{~d},{ }^{2} J_{\mathrm{C}-\mathrm{F}}=22 \mathrm{~Hz}\right), 77.6,76.1(\mathrm{q}$, $\left.{ }^{2} J_{\mathrm{C}-\mathrm{F}}=30.2 \mathrm{~Hz}\right) ;{ }^{19} \mathrm{~F}$ NMR $\left(282 \mathrm{MHz}, \mathrm{CDCl}_{3}\right) \delta:-79.3(\mathrm{~s}, 3 \mathrm{~F}),-111.3(\mathrm{~s}, 1 \mathrm{~F}) ; \mathrm{IR}(\mathrm{KBr}) \mathrm{v}: 3455$, 1383; HRMS (EI $-\mathrm{TOF}$ ): exact mass calcd. for $\mathrm{C}_{9} \mathrm{H}_{7} \mathrm{~F}_{4} \mathrm{NO}_{3}[\mathrm{M}]^{+}: 253.0362$, found 253.0362; the enantiomeric purity of $\mathbf{4 e}$ was determined by chiral HPLC analysis using a Daicel Chiralcel OJ$\mathrm{H}$ column; $n$-hexane/2-propanol 9:1; $1 \mathrm{~mL} / \mathrm{min} ; \lambda=215 \mathrm{~nm}$. $t_{\mathrm{R}}($ major $)=23.0 \mathrm{~min} ; t_{\mathrm{R}}($ minor $)=$ $26.2 \mathrm{~min} ; 97 \%$ ee.

(S)-2-(4-tert-butyl-phenyl)-1,1,1-trifluoro-3-nitro-propan-2-ol (4f):<smiles>CN(C)CC(O)(c1ccc(C(C)(C)C)cc1)C(F)(F)F</smiles>
. $7.45(\mathrm{~d}, J=6.6 \mathrm{~Hz}, 2 \mathrm{H}), 5.09(\mathrm{~d}, J=13.8 \mathrm{~Hz}, 1 \mathrm{H}), 5.00(\mathrm{~d}, J=13.5 \mathrm{~Hz}, 1 \mathrm{H}), 4.59$ $(\mathrm{s}, 1 \mathrm{H}), 1.32(\mathrm{~s}, 9 \mathrm{H}) ;{ }^{13} \mathrm{C}$ NMR $\left(75.5 \mathrm{MHz}, \mathrm{CDCl}_{3}\right) \delta: 153.3,130.2\left(\mathrm{~d},{ }^{3} J_{\mathrm{C}-\mathrm{F}}=1.5 \mathrm{~Hz}\right), 126.3$, 126.0, $123.7\left(\mathrm{q},{ }^{1} J_{\mathrm{C}-\mathrm{F}}=285.4 \mathrm{~Hz}\right), 77.6\left(\mathrm{q},{ }^{2} J_{\mathrm{C}-\mathrm{F}}=30.2 \mathrm{~Hz}\right), 34.8,31.3 ;{ }^{19} \mathrm{~F}$ NMR $(282 \mathrm{MHz}$, $\left.\mathrm{CDCl}_{3}\right) \delta$ : -79.4 (s, 3F); HRMS $\left(\mathrm{FAB}^{+}\right)$: exact mass calcd. for $\mathrm{C}_{13} \mathrm{H}_{16} \mathrm{~F}_{3} \mathrm{NO}_{3}[\mathrm{M}]^{+}:$291.1082, found 291.1089; the enantiomeric purity of $\mathbf{4 f}$ was determined by chiral HPLC analysis using a Daicel Chiralpak AS-H column; $n$-hexane $/ 2$-propanol 99:1; $1 \mathrm{~mL} / \mathrm{min} ; \lambda=215 \mathrm{~nm} . t_{\mathrm{R}}$ (major) $=$ $19.4 \mathrm{~min} ; t_{\mathrm{R}}($ minor $)=22.9 \mathrm{~min} ; 98 \%$ ee . 
(S)-1,1,1-trifluoro-2-nitromethyl-4-phenyl-but-3-yn-2-ol (4g):<smiles>O=[N+]([O-])CC(O)(C#Cc1ccccc1)C(F)(F)F</smiles>

The title compound $\mathbf{4 g}$ was prepared according to the general procedure described in section D. Yield: 82\%; yellow oil; $[\alpha]^{25}+33.8^{\circ}\left(\mathrm{c} 0.74, \mathrm{CHCl}_{3}\right) ;{ }^{1} \mathrm{H}$ NMR (300 $\left.\mathrm{MHz}, \mathrm{CDCl}_{3}\right) \delta:$ 7.50-7.32 (m, 5H), $4.83(\mathrm{~s}, 1 \mathrm{H}) ;{ }^{13} \mathrm{C} \mathrm{NMR}\left(75.5 \mathrm{MHz}, \mathrm{CDCl}_{3}\right) \delta: 132.4,130.3$, $128.7,122.4\left(\mathrm{q},{ }^{1} J_{\mathrm{C}-\mathrm{F}}=286.1 \mathrm{~Hz}\right), 120.1,90.0,79.0,78.2,70.5\left(\mathrm{q},{ }^{2} J_{\mathrm{C}-\mathrm{F}}=28 \mathrm{~Hz}\right) ;{ }^{19} \mathrm{~F}$ NMR $(282$ $\left.\mathrm{MHz}, \mathrm{CDCl}_{3}\right) \delta$ : -80.4 (s, 3F); the enantiomeric purity of $\mathbf{4 f}$ was determined by chiral HPLC analysis using a Daicel Chiralcel OJ-H column; $n$-hexane/2-propanol 9:1; $1 \mathrm{~mL} / \mathrm{min} ; \lambda=215$ nm. $t_{\mathrm{R}}($ major $)=28.6 \mathrm{~min} ; t_{\mathrm{R}}($ minor $)=31.1 \mathrm{~min} ; 80 \%$ ee .

\section{E) General procedure for the "enantiomer self-disproportionation test":}

To a solution of $[(\Delta, S, S, S)-B i n o l a m]_{3} \cdot \operatorname{La}(\mathrm{OTf})_{3} 2 \mathbf{L a}(416 \mathrm{mg}, 0.212 \mathrm{mmol}, 10 \mathrm{~mol} \%$, 0.1 equiv. $)$ in dry acetonitrile $(3.5 \mathrm{~mL})$, at room temperature, under argon, proton sponge $\AA(760$ $\mu \mathrm{L}, 0.212 \mathrm{mmol}, 10 \mathrm{~mol} \%, 0.1$ equiv., $280 \mathrm{mM}$ solution in dry acetonitrile) was added. After stirring for $5 \mathrm{~min}$. 1,1,1-trifluoromethyl-2-butanone 3a $(304 \mu \mathrm{L}, 2.12 \mathrm{mmol}, 1$ equiv.) and excess nitromethane $(1.16 \mathrm{~mL}, 21.2 \mathrm{mmol}, 10$ equiv.) were added and the mixture stirred for 48 $\mathrm{h}$. The reaction mixture was then quenched with the appropriate amount of TFA $(0.424 \mathrm{mmol}$, 0.2 equiv.) followed by evaporation to dryness.

An "enantiomer self-disproportionation test" was run upon the crude material by column chromatography under the following conditions: room temperature; column diameter $\mathrm{d}$ $=1 \mathrm{~cm}$, column length $=20 \mathrm{~cm}$; Merck silica gel $60(70-230 \mathrm{mesh})(6 \mathrm{~g}) ; \mathrm{CHCl}_{3}$ as eluant; volume collected for each fraction: $\mathrm{V}=4 \mathrm{~mL}$. The enantiomeric purity of $\mathbf{4 a}$ was determined by chiral HPLC analysis upon each of the eight fractions collected: Daicel Chiralpak AS-H; $n$ hexane/2-propanol 95:5; $1 \mathrm{~mL} / \mathrm{min}, \lambda=215 \mathrm{~nm}$. The overall yield of $\mathbf{4 a}$ was found to be $98 \%$ (388 mg).

Table 1. Test of self-disproportionation of enantiomers carried out upon compound $\mathbf{4 a}$ ( $52 \%$ ee) on achiral silica gel using $\mathrm{CHCl}_{3}$ as an eluant.

\begin{tabular}{ccc}
\hline Fraction & $\% \mathrm{ee}^{a}$ & Mass \% \\
\hline 1 & - & 0 \\
2 & - & 0.75 \\
3 & 46.98 & 56.1 \\
4 & 51.88 & 76.23 \\
5 & 52.16 & 86.04 \\
6 & 52.96 & 92.08 \\
7 & 55.68 & 95.85 \\
8 & 52.08 & 97.61 \\
\hline
\end{tabular}

${ }^{a}$ Starting $\%$ ee of the whole sample 4a was established to be $52 \%$ ee after purification by column chromatography under the following conditions: Merck silica gel 60 (70-230 mesh) $(6 \mathrm{~g}) ; \mathrm{CHCl}_{3}$ as eluant. The enantiomeric purity of $\mathbf{4 a}$ was determined by chiral HPLC analysis: Daicel Chiralpak AS-H; $n$-hexane/2-propanol 95:5; $1 \mathrm{~mL} / \mathrm{min} ; \lambda=215 \mathrm{~nm}$. 


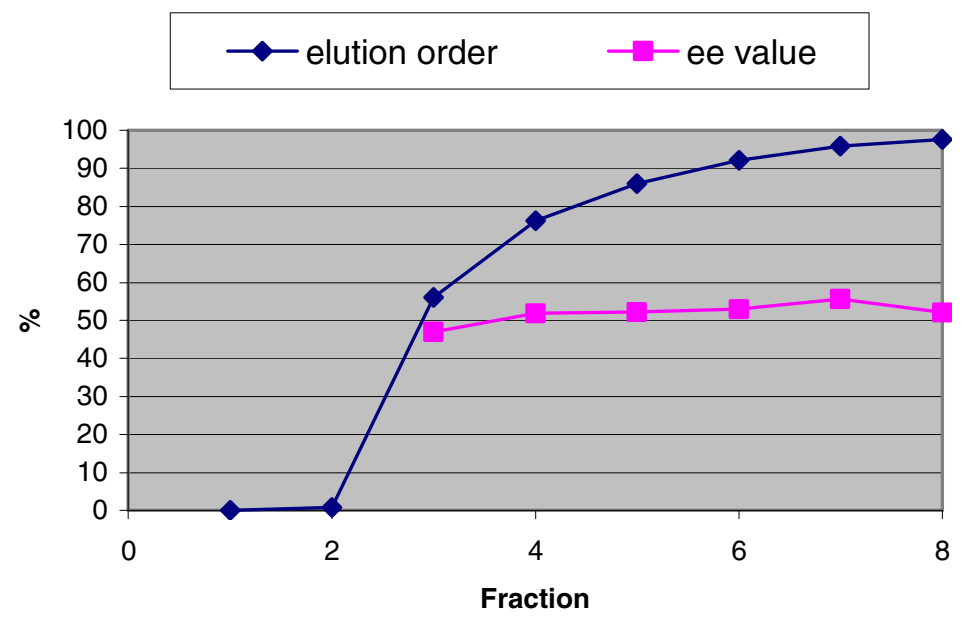

Figure 1. Graphical representation of Table 1.

According with the data collected in Table 1 and represented in Figure 1, we are completely confident that the enantiomeric purity reported herein for compounds $\mathbf{4 a - g}$ and $\mathbf{5 b}$, $\mathbf{5 c}, \mathbf{5 f}, \mathbf{5 g}$ (see below) is absent of the so-called self-disproportionation effect reported by Soloshonok. ${ }^{6}$

F) General procedure for the reduction of $\alpha$-trifluoromethyl tertiary nitroaldols $4 \mathrm{~b}, 4 \mathrm{c}, 4 \mathrm{f}$, $4 \mathrm{~g}$ to the corresponding $\beta$-amino- $\alpha$-trifluoromethyl tertiary alcohols $5 \mathrm{~b}, 5 \mathrm{c}, 5 \mathrm{f}, 5 \mathrm{~g}$ :

To a stirred solution of the corresponding $\alpha$-trifluoromethyl tertiary nitroaldol 4 (1 equiv.) and $\mathrm{NiCl}_{2} \cdot 6 \mathrm{H}_{2} \mathrm{O}$ (1 equiv.) in $\mathrm{MeOH}$ at $0{ }^{\circ} \mathrm{C}, \mathrm{NaBH}_{4}$ (12 equiv.) was added in portions. The reaction mixture was stirred at $0{ }^{\circ} \mathrm{C}$ for $2-3 \mathrm{~h}$. Then the reaction mixture was quenched with a saturated $\mathrm{NH}_{4} \mathrm{Cl}$ solution. The solution was filtered and $\mathrm{MeOH}$ was removed by evaporation under reduced pressure. The remaining aqueous phase was extracted with $\mathrm{CHCl}_{3}$. The combined organic layers were dried over anhydrous $\mathrm{K}_{2} \mathrm{CO}_{3}$, filtered and the solvent removed under reduced pressure. The crude product was purified by column chromatography (silica gel, $\mathrm{CHCl}_{3} / \mathrm{MeOH}$ 5:1) thereby affording the desired product 5.

\section{(S)-2-aminomethyl-1,1,1-trifluoro-3-phenyl-propan-2-ol (5b):}

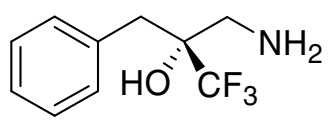

5b The title compound $\mathbf{5 b}$ was prepared according to the general procedure described in section F. Yield: 78\%; white solid; mp: $73-75{ }^{\circ} \mathrm{C}$; $[\alpha]^{25}{ }_{\mathrm{D}}+20^{\circ}\left(\mathrm{c} 0.5, \mathrm{CHCl}_{3}\right) ;{ }^{1} \mathrm{H}$ NMR (300 MHz, $\left.\mathrm{CDCl}_{3}\right) \delta$ : 7.33-7.24 (m, 5H), $3.19(\mathrm{~d}, J=13.7 \mathrm{~Hz}, 1 \mathrm{H}), 3.04(\mathrm{~d}, J=13.5 \mathrm{~Hz}$, $1 \mathrm{H}), 2.71(\mathrm{~d}, J=14.1 \mathrm{~Hz}, 1 \mathrm{H}), 2.58(\mathrm{~d}, J=13.5 \mathrm{~Hz}, 1 \mathrm{H}) ;{ }^{13} \mathrm{C}$ NMR $\left(75.5 \mathrm{MHz}, \mathrm{CDCl}_{3}\right) \delta$ : $134.8,131.2,128.8\left(\mathrm{q},{ }^{1} J_{\mathrm{C}-\mathrm{F}}=287.1 \mathrm{~Hz}\right), 128.3,127.3,72.6\left(\mathrm{q},{ }^{2} J_{\mathrm{C}-\mathrm{F}}=25.8 \mathrm{~Hz}\right), 41.8,38.3 ;{ }^{19} \mathrm{~F}$ NMR (282 MHz, $\left.\mathrm{CDCl}_{3}\right) \delta$ : -80.6 (s, 3F); IR (KBr)v: 3395, 3331; HRMS (ESI ${ }^{+}$TOF): exact mass calcd. for $\mathrm{C}_{10} \mathrm{H}_{13} \mathrm{~F}_{3} \mathrm{NO}[\mathrm{M}+\mathrm{H}]^{+}: 220.0949$, found 220.0944; the enantiomeric purity of $\mathbf{5 b}$ was determined by chiral HPLC analysis using a Daicel Chiralcel OJ-H column; $n$-hexane/2propanol 9:1; $1 \mathrm{~mL} / \mathrm{min} ; \lambda=215 \mathrm{~nm} . t_{\mathrm{R}}($ major $)=10.5 \mathrm{~min} ; t_{\mathrm{R}}($ minor $)=13.2 \mathrm{~min} ; 84 \%$ ee.

(S)-3-amino-1,1,1-trifluoro-2-phenyl-propan-2-ol (5c): ${ }^{7}$ 
<smiles>NCC(O)(c1ccccc1)C(F)(F)F</smiles>

5c The title compound $\mathbf{5 c}$ was prepared according to the general procedure described in section F. Yield: 79\%; white solid; mp: $63-66^{\circ} \mathrm{C}$; $[\alpha]^{25}{ }_{\mathrm{D}}+40.5^{\circ}\left(\mathrm{c} 0.765, \mathrm{CHCl}_{3}\right)$; ${ }^{1} \mathrm{H} \mathrm{NMR}\left(300 \mathrm{MHz}, \mathrm{CDCl}_{3}\right) \delta: 7.58(\mathrm{~d}, J=6.9 \mathrm{~Hz}, 2 \mathrm{H}), 7.40-7.37(\mathrm{~m}, 3 \mathrm{H}), 5.44$ (br s, $\left.1 \mathrm{H}\right), 3.56$ $(\mathrm{d}, J=13.2 \mathrm{~Hz}, 1 \mathrm{H}), 3.03(\mathrm{~d}, J=13.2 \mathrm{~Hz}, 1 \mathrm{H}), 1.35(\mathrm{br} \mathrm{s}, 2 \mathrm{H}) ;{ }^{13} \mathrm{C}$ NMR $\left(75.5 \mathrm{MHz}, \mathrm{CDCl}_{3}\right) \delta$ : 137.6, 128.6, 128.5, 126.3, $125.9\left(\mathrm{q},{ }^{1} J_{\mathrm{C}-\mathrm{F}}=286.1 \mathrm{~Hz}\right), 74.0\left(\mathrm{q},{ }^{2} J_{\mathrm{C}-\mathrm{F}}=29.4 \mathrm{~Hz}\right), 45.6 ;{ }^{19} \mathrm{~F} \mathrm{NMR}$ $\left(282 \mathrm{MHz}, \mathrm{CDCl}_{3}\right) \delta$ : -78.7 (s, 3F); IR (KBr)v: 3388, 3347; HRMS (ESI ${ }^{+}-\mathrm{TOF}$ ): exact mass calcd. for $\mathrm{C}_{9} \mathrm{H}_{11} \mathrm{~F}_{3} \mathrm{NO}[\mathrm{M}+\mathrm{H}]^{+}: 206.0792$, found 206.0786; the enantiomeric purity of $\mathbf{5 c}$ was determined by chiral HPLC analysis using a Daicel Chiralcel OJ-H column; $n$-hexane/2propanol 9:1; $1 \mathrm{~mL} / \mathrm{min} ; \lambda=215 \mathrm{~nm} . t_{\mathrm{R}}($ major $)=10.2 \mathrm{~min} ; t_{\mathrm{R}}(\operatorname{minor})=17.8 \mathrm{~min} ; 94.5 \%$ ee.

(S)-3-amino-2-(4-tert-butyl-phenyl)-1,1,1-trifluoro-propan-2-ol (5f):<smiles>CC(C)(C)c1ccc(C(O)(CN)C(F)(F)F)cc1</smiles>

$5 \mathbf{f} \quad$ The title compound $\mathbf{5 f}$ was prepared according to the general procedure described in section F. Yield: 87\%; white solid; mp: 89-92 ${ }^{\circ} \mathrm{C}$; $[\alpha]^{25}{ }_{\mathrm{D}}+28^{\circ}\left(\mathrm{c} 0.5, \mathrm{CHCl}_{3}\right) ;{ }^{1} \mathrm{H}$ NMR $\left(300 \mathrm{MHz}, \mathrm{CDCl}_{3}\right) \delta: 7.49(\mathrm{~d}, J=8.4 \mathrm{~Hz}, 2 \mathrm{H}), 7.40(\mathrm{~d}, J=8.7 \mathrm{~Hz}, 2 \mathrm{H}), 3.52(\mathrm{~d}, J=12$ $\mathrm{Hz}, 1 \mathrm{H}), 3.08(\mathrm{~d}, J=12 \mathrm{~Hz}, 1 \mathrm{H}), 1.33(\mathrm{~s}, 9 \mathrm{H}) ;{ }^{13} \mathrm{C} \mathrm{NMR}\left(75.5 \mathrm{MHz}, \mathrm{CDCl}_{3}\right) \delta: 151.6,134.5$, $126.0,125.7\left(\mathrm{q},{ }^{1} J_{\mathrm{C}-\mathrm{F}}=243.1 \mathrm{~Hz}\right), 125.4,74.5\left(\mathrm{q},{ }^{2} J_{\mathrm{C}-\mathrm{F}}=27.9 \mathrm{~Hz}\right), 45.6,34.7,31.4 ;{ }^{19} \mathrm{~F}$ NMR $\left(282 \mathrm{MHz}, \mathrm{CDCl}_{3}\right) \delta$ : -78.8 (s, 3F); IR (KBr)v: 3375, 3310; HRMS (ESI'-TOF): exact mass calcd. for $\mathrm{C}_{13} \mathrm{H}_{19} \mathrm{~F}_{3} \mathrm{NO}[\mathrm{M}+\mathrm{H}]^{+}: 262.1418$, found 262.1413 ; the enantiomeric purity of $\mathbf{5 f}$ was determined by chiral HPLC analysis using a Daicel Chiralcel OJ-H column; $n$-hexane/2propanol 9:1; $1 \mathrm{~mL} / \mathrm{min} ; \lambda=215 \mathrm{~nm} . t_{\mathrm{R}}($ major $)=12.9 \mathrm{~min} ; t_{\mathrm{R}}($ minor $)=16.7 \mathrm{~min} ; 97 \%$ ee.

\section{(S)-2-aminomethyl-1,1,1-trifluoro-4-phenyl-butan-2-ol (5g):}<smiles>NC[C@@](O)(CCc1ccccc1)C(F)(F)F</smiles>
2.77-2.62 (m, 2H), 2.17-2.01 (m, 1H), 1.83-1.73 (m, 1H); ${ }^{13} \mathrm{C}$ NMR $\left(75.5 \mathrm{MHz}, \mathrm{CDCl}_{3}\right) \delta$ : $141.7,129.0,128.5,123.2\left(\mathrm{q},{ }^{1} J_{\mathrm{C}-\mathrm{F}}=286.9 \mathrm{~Hz}\right), 72.2\left(\mathrm{q},{ }^{2} J_{\mathrm{C}-\mathrm{F}}=26.4 \mathrm{~Hz}\right), 42.9,35.0,29.0 ;{ }^{19} \mathrm{~F}$ NMR (282 MHz, CDCl $)$ ) $:-80.1$ (s, 3F); HRMS (ESI ${ }^{+}$-TOF): exact mass calcd. for $\mathrm{C}_{11} \mathrm{H}_{15} \mathrm{~F}_{3} \mathrm{NO}$ $[\mathrm{M}+\mathrm{H}]^{+}: 234.1105$, found 234.1100 ; the enantiomeric purity of $\mathbf{5 g}$ was determined by chiral HPLC analysis using a Daicel Chiralpak AS-H column; $n$-hexane/2-propanol 9:1; $1 \mathrm{~mL} / \mathrm{min} ; \lambda$ $=215 \mathrm{~nm} . t_{\mathrm{R}}($ minor $)=10.6 \mathrm{~min} ; t_{\mathrm{R}}($ major $)=11.8 \mathrm{~min} ; 72 \%$ ee.

\section{G) Attempted kinetic resolution of racemic nitroaldol 4c:}

To a solution of $[(\Delta, S, S, S) \text {-Binolam }]_{3} \cdot \mathrm{La}(\mathrm{OTf})_{3} \mathbf{2} \mathbf{L a}(260 \mathrm{mg}, 0.1325 \mathrm{mmol}, 25 \mathrm{~mol} \%$, 0.25 equiv. $)$ in dry acetonitrile $(5 \mathrm{~mL})$, at $-40{ }^{\circ} \mathrm{C}$, under argon, proton sponge ${ }^{\circ}(475 \mu \mathrm{L}, 0.1325$ $\mathrm{mmol}, 25 \mathrm{~mol} \%, 0.25$ equiv., $280 \mathrm{mM}$ solution in dry acetonitrile) was added. After stirring for 5 min. $(R, S)$-1,1,1-trifluoro-3-nitro-2-phenyl-propan-2-ol $4 \mathbf{c}(125 \mathrm{mg}, 0.53 \mathrm{mmol}, 1$ equiv.) and excess nitromethane $(291 \mu \mathrm{L}, 5.3 \mathrm{mmol}, 10$ equiv.) were added and the mixture stirred for $96 \mathrm{~h}$. The reaction mixture was then quenched with the appropriate amount of TFA $(0.265 \mathrm{mmol}$, 0.50 equiv.) followed by evaporation to dryness. The crude mixture was purified by column chromatography (silica gel, $\mathrm{CHCl}_{3}$ ) thereby affording $\alpha$-trifluoromethyl tertiary nitroaldol $\mathbf{4 c}$ 
(76\% recovered yield). The enantiomeric purity ( $0 \%$ ee) of $\mathbf{4 c}$ was determined by chiral HPLC analysis: Daicel Chiralcel OJ-H; $n$-hexane/2-propanol 9:1; $1 \mathrm{~mL} / \mathrm{min}, \lambda=215 \mathrm{~nm}$.

\section{H) Evaluation of retro-nitroaldol reaction upon enantiomerically enriched 4c:}

To a solution of $[(\Delta, S, S, S) \text {-Binolam }]_{3} \cdot \mathrm{La}(\mathrm{OTf})_{3} 2 \mathbf{L a}(216 \mathrm{mg}, 0.11 \mathrm{mmol}, 25 \mathrm{~mol} \%$, 0.25 equiv.) in dry acetonitrile $(5 \mathrm{~mL})$, at $-40{ }^{\circ} \mathrm{C}$, under argon, proton sponge ${ }^{\circledR}(394 \mu \mathrm{L}, 0.11$ mmol, $25 \mathrm{~mol} \%, 0.25$ equiv., $280 \mathrm{mM}$ solution in dry acetonitrile) was added. After stirring for 5 min. (S)-1,1,1-trifluoro-3-nitro-2-phenyl-propan-2-ol 4c (96\% ee) (104 mg, $0.441 \mathrm{mmol}, 1$ equiv.) was added and the mixture stirred for $96 \mathrm{~h}$. The reaction mixture was then quenched with the appropriate amount of TFA $(0.265 \mathrm{mmol}, 0.50$ equiv. $)$ followed by evaporation to dryness. The crude mixture was purified by column chromatography (silica gel, $\mathrm{CHCl}_{3}$ ) thereby affording $\alpha$-trifluoromethyl tertiary nitroaldol $4 \mathbf{c}$ (99\% recovered yield). The enantiomeric excess $(96 \%$ ee) of $\mathbf{4 c}$ was determined by chiral HPLC analysis: Daicel Chiralcel OJ-H; $n$ hexane/2-propanol 9:1; $1 \mathrm{~mL} / \mathrm{min}, \lambda=215 \mathrm{~nm}$.

\section{I) General procedure for the synthesis of racemic $\alpha$-trifluoromethyl tertiary nitroaldols 4a-g:}

To a stirred solution of trifluoromethyl ketone 3a-g (0.02 mol, 1 equiv.) and excess nitromethane $(11 \mathrm{~mL}, 0.2 \mathrm{~mol}, 10$ equiv. $)$ in dichloromethane $(10 \mathrm{~mL})$ at $0{ }^{\circ} \mathrm{C}$, proton sponge ${ }^{\circledR}$ (428 mg, $0.002 \mathrm{~mol}, 10 \mathrm{~mol} \%, 0.1$ equiv.) was added and the mixture stirred at room temperature until no more nitroaldol adduct was formed (reaction monitorized by TLC). The reaction mixture was then quenched with $\mathrm{HCl} 5 \%(10 \mathrm{~mL})$. After the phases separate, the organic layer was washed with water until neutral $\mathrm{pH}$ and with brine $(10 \mathrm{~mL})$. The organic layer was dried over $\mathrm{Na}_{2} \mathrm{SO}_{4}$ followed by evaporation under reduced pressure thereby affording the corresponding $\alpha$-trifluoromethyl tertiary nitroaldol $\mathbf{4 a - g}$. The crude product was purified by column chromatography on a silica gel column using $n$-hexane polarized gradually with chloroform as eluant affording $\alpha$-trifluoromethyl tertiary nitroaldols $\mathbf{4 a - g}$ in $41-88 \%$ yield.

\section{J) Assignment of the absolute configuration of adduct $5 \mathrm{c}$ :}

X-ray data on 5c were collected on a Bruker Nonius FR591 CCD2000 diffractometer at 100 K. Data reduction were performed with the HKL Denzo (Otwinowski, Z. \& Minor, W., 1997), x2sad, SADABS, XPREP (Sheldrick, G., 1997-2007), twinlaw and hklf5 (Bolte, M., 2004) software packages . The structure was solved by direct methods SIR-97 (Altomare. A. et al., 1999) and refined by full-matrix least-squares using SHELX-97 (Sheldrick, G., 1997) with anisotropic displacement for all non-hydrogen atoms. Hydrogen atoms were inserted in calculated positions with isotropic displacement parameters proportional to those of their neighboring atoms except those attached to the hydroxyl and amino groups that were found in the corresponding Fourier difference synthesis and refined as isotropic. Atomic scattering factors were taken from International Tables for X-ray crystallography; Wilson, A. J. C., Ed.; Kluwer Academic Publishers: Dordrecht, 1992. Diagrams of the molecular structure were produced by the ORTEP-3 for Windows program (Farrugia, L., 1997). Crystallographic data for 5c have been deposited at the Cambridge Crystallographic Data Centre and allocated the deposition number CCDC 662278. 


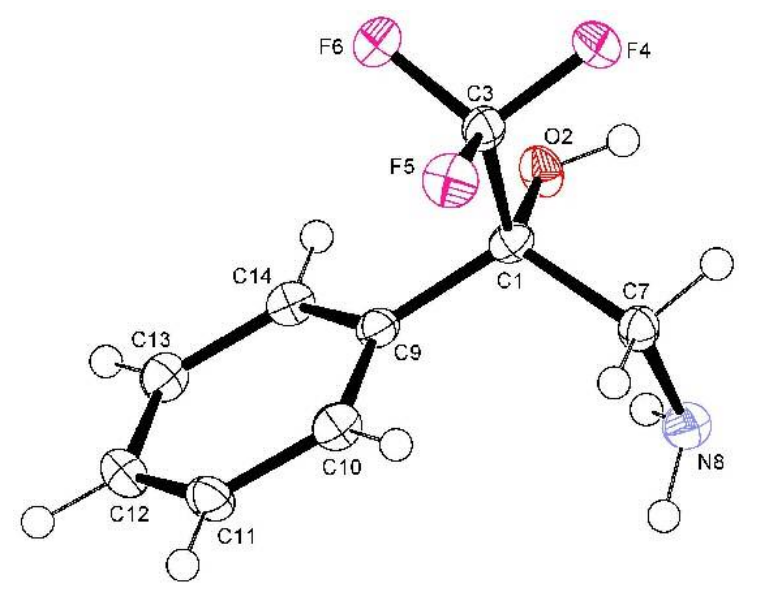

Figure 2. X-ray crystallographic structure of $(S)-5$ c. Ellipsoids drawn at the 50\% probability level.

Table 2. Crystal data and structure refinement for $(S)-\mathbf{5 c}$.

Identification code

Empirical formula

Formula weight

Temperature

Wavelength

Crystal system

Space group

Unit cell dimensions

Volume

$\mathrm{Z}$

Density (calculated)

Absorption coefficient

$\mathrm{F}(000)$

Crystal size

Theta range for data collection

Index ranges

Reflections collected

Independent reflections

Completeness to theta $=65.25^{\circ}$

Absorption correction

Max. and min. transmission

Refinement method

Data / restraints / parameters

Goodness-of-fit on $\mathrm{F}^{2}$
$2007 \mathrm{~ms} 02$

$\mathrm{C}_{9} \mathrm{H}_{10} \mathrm{~F}_{3} \mathrm{NO}$

205.18

100.0(1) K

$1.54178 \AA$

Monoclinic

$P 2_{1}$

$a=7.2483(4) \AA$ $\alpha=90^{\circ}$

$b=5.7003(3) \AA$ $\beta=94.877(3)^{\circ}$

$c=10.8181(6) \AA$ $\gamma=90^{\circ}$

445.36(4) $\AA^{3}$

2

\section{$1.530 \mathrm{Mg} / \mathrm{m}^{3}$}

$1.251 \mathrm{~mm}^{-1}$

212

$0.38 \times 0.06 \times 0.01 \mathrm{~mm}^{3}$

4.1 to $65.25^{\circ}$

$-8<=\mathrm{h}<=8,-6<=\mathrm{k}<=6,-12<=\mathrm{l}<=12$

17879

$1525[\mathrm{R}(\mathrm{int})=0.0596]$

$99.8 \%$

Semi-empirical from equivalents

0.9844 and 0.7488

Full-matrix least-squares on $\mathrm{F}^{2}$

$1525 / 1 / 137$

1.131 
Twin law (reciprocal space):

Twin domains ratio (\%):

Final $\mathrm{R}$ indices $[\mathrm{I}>2 \operatorname{sigma}(\mathrm{I})]$

$\mathrm{R}$ indices (all data)

Absolute structure parameter (Flack)

Largest diff. peak and hole

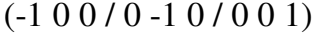

$$
\begin{aligned}
& \text { 56.4(1)/43.6(1) } \\
& \mathrm{R} 1=0.044, \mathrm{wR} 2=0.1221 \\
& \mathrm{R} 1=0.045, \mathrm{wR}_{2}=0.1236 \\
& \text { 0.01(16) } \\
& 0.203 \text { and }-0.266 \text { e. } \AA^{-3}
\end{aligned}
$$

Table 3. Atomic coordinates ( $\mathrm{x} 10^{4}$ ) and equivalent isotropic displacement parameters $\left(\AA^{2} \times 10^{3}\right)$ for $(S)$-5c. U(eq) is defined as one third of the trace of the orthogonalized $\mathrm{U}^{\mathrm{ij}}$ tensor.

\begin{tabular}{lcccc}
\hline & $\mathrm{x}$ & $\mathrm{y}$ & $\mathrm{z}$ & $\mathrm{U}(\mathrm{eq})$ \\
\hline $\mathrm{C}(1)$ & $2624(3)$ & $2741(4)$ & $3241(2)$ & $17(1)$ \\
$\mathrm{O}(2)$ & $3833(2)$ & $4576(3)$ & $3674(1)$ & $21(1)$ \\
$\mathrm{C}(3)$ & $675(3)$ & $3455(4)$ & $3543(2)$ & $19(1)$ \\
$\mathrm{F}(4)$ & $570(2)$ & $3731(2)$ & $4777(1)$ & $25(1)$ \\
$\mathrm{F}(5)$ & $-641(2)$ & $1888(2)$ & $3159(1)$ & $25(1)$ \\
$\mathrm{F}(6)$ & $149(2)$ & $5536(2)$ & $3023(1)$ & $26(1)$ \\
$\mathrm{C}(7)$ & $3151(3)$ & $462(4)$ & $3933(2)$ & $18(1)$ \\
$\mathrm{N}(8)$ & $5111(3)$ & $-159(4)$ & $3853(2)$ & $22(1)$ \\
$\mathrm{C}(9)$ & $2638(3)$ & $2561(4)$ & $1836(2)$ & $17(1)$ \\
$\mathrm{C}(10)$ & $1829(3)$ & $657(4)$ & $1191(2)$ & $21(1)$ \\
$\mathrm{C}(11)$ & $1751(3)$ & $577(4)$ & $-101(2)$ & $22(1)$ \\
$\mathrm{C}(12)$ & $2490(3)$ & $2382(5)$ & $-751(2)$ & $22(1)$ \\
$\mathrm{C}(13)$ & $3319(3)$ & $4263(4)$ & $-122(2)$ & $21(1)$ \\
$\mathrm{C}(14)$ & $3381(3)$ & $4367(4)$ & $1171(2)$ & $19(1)$ \\
& & & & \\
\hline
\end{tabular}

Table 4. Bond lengths $[\AA]$ and angles $\left[{ }^{\circ}\right]$ for $(S)-5$ c.

\begin{tabular}{ll}
\hline $\mathrm{C}(1)-\mathrm{O}(2)$ & $1.418(3)$ \\
$\mathrm{C}(1)-\mathrm{C}(9)$ & $1.524(3)$ \\
$\mathrm{C}(1)-\mathrm{C}(7)$ & $1.532(3)$ \\
$\mathrm{C}(1)-\mathrm{C}(3)$ & $1.532(3)$ \\
$\mathrm{O}(2)-\mathrm{H}(2)$ & $0.84(3)$ \\
$\mathrm{C}(3)-\mathrm{F}(5)$ & $1.347(2)$ \\
$\mathrm{C}(3)-\mathrm{F}(4)$ & $1.353(2)$ \\
$\mathrm{C}(3)-\mathrm{F}(6)$ & $1.354(3)$ \\
$\mathrm{C}(7)-\mathrm{N}(8)$ & $1.474(3)$
\end{tabular}




\begin{tabular}{|c|c|}
\hline $\mathrm{C}(7)-\mathrm{H}(7 \mathrm{~A})$ & 0.99 \\
\hline $\mathrm{C}(7)-\mathrm{H}(7 \mathrm{~B})$ & 0.99 \\
\hline $\mathrm{N}(8)-\mathrm{H}(8 \mathrm{~A})$ & $0.92(3)$ \\
\hline $\mathrm{N}(8)-\mathrm{H}(8 \mathrm{~B})$ & $0.93(3)$ \\
\hline $\mathrm{C}(9)-\mathrm{C}(14)$ & $1.390(3)$ \\
\hline $\mathrm{C}(9)-\mathrm{C}(10)$ & $1.392(3)$ \\
\hline $\mathrm{C}(10)-\mathrm{C}(11)$ & $1.394(3)$ \\
\hline $\mathrm{C}(10)-\mathrm{H}(10)$ & 0.95 \\
\hline $\mathrm{C}(11)-\mathrm{C}(12)$ & $1.380(3)$ \\
\hline $\mathrm{C}(11)-\mathrm{H}(11)$ & 0.95 \\
\hline $\mathrm{C}(12)-\mathrm{C}(13)$ & $1.380(3)$ \\
\hline $\mathrm{C}(12)-\mathrm{H}(12)$ & 0.95 \\
\hline $\mathrm{C}(13)-\mathrm{C}(14)$ & $1.397(3)$ \\
\hline $\mathrm{C}(13)-\mathrm{H}(13)$ & 0.95 \\
\hline $\mathrm{C}(14)-\mathrm{H}(14)$ & 0.95 \\
\hline $\mathrm{O}(2)-\mathrm{C}(1)-\mathrm{C}(9)$ & $108.82(16)$ \\
\hline $\mathrm{O}(2)-\mathrm{C}(1)-\mathrm{C}(7)$ & $110.07(16)$ \\
\hline $\mathrm{C}(9)-\mathrm{C}(1)-\mathrm{C}(7)$ & $114.08(17)$ \\
\hline $\mathrm{O}(2)-\mathrm{C}(1)-\mathrm{C}(3)$ & $106.77(17)$ \\
\hline $\mathrm{C}(9)-\mathrm{C}(1)-\mathrm{C}(3)$ & $108.34(16)$ \\
\hline $\mathrm{C}(7)-\mathrm{C}(1)-\mathrm{C}(3)$ & $108.49(17)$ \\
\hline $\mathrm{C}(1)-\mathrm{O}(2)-\mathrm{H}(2)$ & $105(2)$ \\
\hline $\mathrm{F}(5)-\mathrm{C}(3)-\mathrm{F}(4)$ & $106.48(16)$ \\
\hline$F(5)-C(3)-F(6)$ & $106.62(16)$ \\
\hline $\mathrm{F}(4)-\mathrm{C}(3)-\mathrm{F}(6)$ & $105.70(17)$ \\
\hline $\mathrm{F}(5)-\mathrm{C}(3)-\mathrm{C}(1)$ & $113.55(17)$ \\
\hline $\mathrm{F}(4)-\mathrm{C}(3)-\mathrm{C}(1)$ & $111.83(17)$ \\
\hline $\mathrm{F}(6)-\mathrm{C}(3)-\mathrm{C}(1)$ & $112.13(17)$ \\
\hline $\mathrm{N}(8)-\mathrm{C}(7)-\mathrm{C}(1)$ & $112.10(18)$ \\
\hline $\mathrm{N}(8)-\mathrm{C}(7)-\mathrm{H}(7 \mathrm{~A})$ & 109.2 \\
\hline $\mathrm{C}(1)-\mathrm{C}(7)-\mathrm{H}(7 \mathrm{~A})$ & 109.2 \\
\hline $\mathrm{N}(8)-\mathrm{C}(7)-\mathrm{H}(7 \mathrm{~B})$ & 109.2 \\
\hline $\mathrm{C}(1)-\mathrm{C}(7)-\mathrm{H}(7 \mathrm{~B})$ & 109.2 \\
\hline $\mathrm{H}(7 \mathrm{~A})-\mathrm{C}(7)-\mathrm{H}(7 \mathrm{~B})$ & 107.9 \\
\hline $\mathrm{C}(7)-\mathrm{N}(8)-\mathrm{H}(8 \mathrm{~A})$ & $113.7(18)$ \\
\hline $\mathrm{C}(7)-\mathrm{N}(8)-\mathrm{H}(8 \mathrm{~B})$ & $107.4(19)$ \\
\hline $\mathrm{H}(8 \mathrm{~A})-\mathrm{N}(8)-\mathrm{H}(8 \mathrm{~B})$ & $109(2)$ \\
\hline
\end{tabular}




$\begin{array}{ll}\mathrm{C}(14)-\mathrm{C}(9)-\mathrm{C}(10) & 118.90(18) \\ \mathrm{C}(14)-\mathrm{C}(9)-\mathrm{C}(1) & 120.11(18) \\ \mathrm{C}(10)-\mathrm{C}(9)-\mathrm{C}(1) & 120.92(19) \\ \mathrm{C}(9)-\mathrm{C}(10)-\mathrm{C}(11) & 120.5(2) \\ \mathrm{C}(9)-\mathrm{C}(10)-\mathrm{H}(10) & 119.8 \\ \mathrm{C}(11)-\mathrm{C}(10)-\mathrm{H}(10) & 119.8 \\ \mathrm{C}(12)-\mathrm{C}(11)-\mathrm{C}(10) & 120.1(2) \\ \mathrm{C}(12)-\mathrm{C}(11)-\mathrm{H}(11) & 119.9 \\ \mathrm{C}(10)-\mathrm{C}(11)-\mathrm{H}(11) & 119.9 \\ \mathrm{C}(13)-\mathrm{C}(12)-\mathrm{C}(11) & 119.98(19) \\ \mathrm{C}(13)-\mathrm{C}(12)-\mathrm{H}(12) & 120 \\ \mathrm{C}(11)-\mathrm{C}(12)-\mathrm{H}(12) & 120 \\ \mathrm{C}(12)-\mathrm{C}(13)-\mathrm{C}(14) & 120.2(2) \\ \mathrm{C}(12)-\mathrm{C}(13)-\mathrm{H}(13) & 119.9 \\ \mathrm{C}(14)-\mathrm{C}(13)-\mathrm{H}(13) & 119.9 \\ \mathrm{C}(9)-\mathrm{C}(14)-\mathrm{C}(13) & 120.36(19) \\ \mathrm{C}(9)-\mathrm{C}(14)-\mathrm{H}(14) & 119.8 \\ \mathrm{C}(13)-\mathrm{C}(14)-\mathrm{H}(14) & 119.8 \\ \end{array}$

Table 5. Anisotropic displacement parameters $\left(\AA^{2} \mathrm{x} 10^{3}\right)$ for $(S)-5 \mathbf{c}$. The anisotropic displacement factor exponent takes the form: $-2 \pi^{2}\left[h^{2} a^{* 2} U^{11}+\ldots+2 h k a^{*} b^{*} U^{12}\right]$

\begin{tabular}{lllllll}
\hline & $\mathrm{U}^{11}$ & $\mathrm{U}^{22}$ & $\mathrm{U}^{33}$ & $\mathrm{U}^{23}$ & $\mathrm{U}^{13}$ & $\mathrm{U}^{12}$ \\
\hline $\mathrm{C}(1)$ & $16(1)$ & $14(1)$ & $21(1)$ & $-1(1)$ & $0(1)$ & $-1(1)$ \\
$\mathrm{O}(2)$ & $26(1)$ & $18(1)$ & $17(1)$ & $-1(1)$ & $-2(1)$ & $-7(1)$ \\
$\mathrm{C}(3)$ & $22(1)$ & $16(1)$ & $18(1)$ & $1(1)$ & $1(1)$ & $-1(1)$ \\
$\mathrm{F}(4)$ & $29(1)$ & $29(1)$ & $19(1)$ & $-2(1)$ & $5(1)$ & $5(1)$ \\
$\mathrm{F}(5)$ & $19(1)$ & $28(1)$ & $29(1)$ & $-1(1)$ & $2(1)$ & $-3(1)$ \\
$\mathrm{F}(6)$ & $28(1)$ & $24(1)$ & $25(1)$ & $3(1)$ & $0(1)$ & $7(1)$ \\
$\mathrm{C}(7)$ & $20(1)$ & $16(1)$ & $17(1)$ & $2(1)$ & $0(1)$ & $-1(1)$ \\
$\mathrm{N}(8)$ & $22(1)$ & $22(1)$ & $23(1)$ & $-1(1)$ & $2(1)$ & $2(1)$ \\
$\mathrm{C}(9)$ & $16(1)$ & $16(1)$ & $19(1)$ & $-1(1)$ & $0(1)$ & $2(1)$ \\
$\mathrm{C}(10)$ & $20(1)$ & $19(1)$ & $24(1)$ & $1(1)$ & $2(1)$ & $-2(1)$ \\
$\mathrm{C}(11)$ & $24(1)$ & $22(1)$ & $20(1)$ & $-6(1)$ & $-1(1)$ & $-1(1)$ \\
$\mathrm{C}(12)$ & $21(1)$ & $27(1)$ & $17(1)$ & $-3(1)$ & $2(1)$ & $-2(1)$ \\
$\mathrm{C}(13)$ & $20(1)$ & $21(1)$ & $23(1)$ & $3(1)$ & $4(1)$ & $-1(1)$ \\
$\mathrm{C}(14)$ & $17(1)$ & $17(1)$ & $23(1)$ & $0(1)$ & $2(1)$ & $-1(1)$
\end{tabular}


The absolute configuration of $\mathbf{5 c}$ was established to be $(S)$ on the basis of the absolute structure parameter (Flack) found in this analysis (0.01(16)). This confirms the initial assignment we had made by comparison of the optical rotation of $5 \mathbf{c}\left([\alpha]^{25}{ }_{D}+40.5^{\circ}(\mathrm{c} 0.765\right.$, $\left.\mathrm{CHCl}_{3}\right)$ ) with the reported data for a partially enriched sample of $(R)-5 \mathbf{c}\left(\mathrm{lit}^{7 \mathrm{a}}[\alpha]^{18}{ }_{\mathrm{D}}-15.1^{\circ},(\mathrm{c}\right.$ $\left.0.762, \mathrm{CHCl}_{3}\right)$ ). As illustrated above, $\mathbf{5 c}$ was obtained by nickel-boride reduction of $\mathbf{4 c}$, following the general reduction procedure shown in section F. In addition, chiral HPLC showed no erosion of the enantiomeric purity in going from $\mathbf{4 c}$ to $\mathbf{5 c}$. Accordingly, the absolute configuration of $\mathbf{4 c}$ can also be assigned to be $(S)$.

${ }^{1}$ Creary, X. J. Org. Chem. 1987, 52, 5026-5030.

${ }^{2}$ Perrin, D.; Armarego, W. L. F.; Perrin, D. R. Purification of Laboratory Chemicals, 2nd Ed.; Pergamon Press: New York, 1980.

3 (a) Simchen, G.; Schmidt, A. Synthesis 1996, 1093-1094. (b) Konno, T.; Takehana, T.; Mishima, M.; Ishihara, T. J. Org. Chem. 2006, 71, 3545-3550.

${ }^{4}$ Singh, R. P.; Cao, G.; Kirchmeier, R. L.; Shreeve, J. M. J. Org. Chem. 1999, 64, 2873-2876.

${ }^{5}$ Misumi, Y.; Bulman, R. A.; Matsumoto, K. Heterocycles 2002, 56, 599-605.

${ }^{6}$ (a) Soloshonok, V. A. Angew. Chem., Int. Ed. 2006, 45, 766-769. (b) Soloshonok, V. A.; Berbasov, D. O. J. Fluorine Chem. 2006, 127, 597-603.

7 (a) Mioskowski, C.; Solladie, G. Tetrahedron 1973, 29, 3669-3674. (b) Schenk, H. A.; Lenkowski, P. W.; Choudhury-Mukherjee, I.; Ko, S.-H.; Stables, J. P.; Patel, M. J.; Brown, M. L. Bioorg. Med. Chem. 2004, 12, 979-993. (c) Choudhury-Mukherjee, I.; Schenk, H. A.; Cechova, S.; Pajewski, T. N.; Kapur, J.; Ellena, J.; Cafiso, D. S.; Brown, M. L. J. Med. Chem. 2003, 46, 2494-2501. 